\title{
Crack tip blunting and cleavage under dynamic conditions
}

\author{
V. P. Rajan and W. A. Curtin ${ }^{1}$ \\ ${ }^{1}$ Institute of Mechanical Engineering, École Polytechnique Fédérale de Lausanne, \\ Lausanne, CH-1015, Switzerland
}

\begin{abstract}
In structural materials with both brittle and ductile phases, cracks often initiate within the brittle phase and propagate dynamically towards the ductile phase. The macroscale, quasistatic toughness of the material thus depends on the outcome of this microscale, dynamic process. Indeed, dynamics has been hypothesized to suppress dislocation emission, which may explain the occurrence of brittle transgranular fracture in mild steels at low temperatures [1]. Here, crack tip blunting and cleavage under dynamic conditions is explored using continuum mechanics and molecular dynamics simulations. The focus is on two questions: 1) whether dynamics can affect the energy barriers for dislocation emission and cleavage, and 2) what happens in the dynamic "overloaded" situation, in which both processes are energetically possible. In either case, dynamics may shift the balance between brittle cleavage and ductile blunting, thereby affecting the intrinsic ductility of the material. To explore these effects in simulation, a novel interatomic potential is used for which the intrinsic ductility is tunable, and a novel simulation technique is employed, termed a "dynamic cleavage test," in which cracks can be run dynamically at a prescribed energy release rate into a material. Both theory and simulation reveal, however, that the intrinsic ductility of a material is unaffected by dynamics. The energy barrier to dislocation emission appears to be identical in quasi-static and dynamic conditions, and, in the overloaded situation, dislocation emission is kinetically preferred. Thus, dynamics cannot embrittle a ductile material, and the origin of brittle failure in certain alloys (e.g., mild steels) appears to be unrelated to dynamic effects at the crack tip.
\end{abstract}

\section{Introduction}

Tough structural metals are often composed of a ductile matrix phase and brittle inclusions or precipitates. Cracks initiate in the brittle phase and should be arrested when 
they encounter the ductile phase. Since such cracks run dynamically in the brittle phase after initiating from pre-existing flaws, the dynamic response of the system may govern the macroscopic "quasi-static" toughness [2,3]. One important example is mild steel, where cracks develop within grain boundary carbides and propagate dynamically toward the primary $\alpha$-ferrite grains. At sufficiently low temperatures, these cracks are observed to cleave through the ferrite, resulting in an undesirable, low-toughness, transgranular mode of failure [1]. Researchers have speculated that dynamic effects are responsible for the propagation of the crack into the ductile matrix, suggesting that, although a stationary crack in the ferrite might emit dislocations and blunt, a running crack may be stabilized against dislocation emission [1]. In other words, dynamics is postulated to alter the competition between the brittle (Griffith cleavage) and ductile (dislocation emission/crack blunting) response. Aside from the engineering problem of practical relevance, a fundamental question is how a dynamic crack behaves when the energy release rate $G$ or stress intensity factor $K$ exceeds both the Griffith cleavage value and the dislocation emission value. Is it possible that cleavage is preferred over dislocation emission in such an "overloaded" dynamic condition, even though dislocation emission is preferred under quasistatic conditions?

There is no theoretical basis for the supposition that a dynamic crack prefers to cleave rather than to blunt. Lin and Thomson [4] analyzed the role of dynamics in the context of the classical Rice-Thomson analysis [5] in mode III loading, and found that dynamic effects were negligible when the slip plane was inclined relative to the crack plane. Furthermore, as we show in Section 2, the more rigorous, energy-based analysis of Rice [6] yields a similar conclusion in mode II: the criterion for dynamic dislocation emission is identical to that for quasi-static emission. Thus, even when its speed is nonzero, the energy barrier to dislocation emission from a crack tip is $G=\gamma_{u s}$, where $\gamma_{u s}$ is the unstable stacking fault energy for slip [6]. However, it is also possible that atomic-scale effects are important. It is well-known that continuum fracture mechanics is unable to explain many important fracture phenomena, including lattice trapping [7-9], crack tip instabilities [10-13], and crack velocities in steady-state [14], all of which depend intimately on the details of bonding between atoms [15]. Atomistic simulations have therefore become increasingly popular for studying crack tip deformation mechanisms and their implications for ductility [16], both in quasi-static [17-20] and dynamic [21-24] conditions. In the latter case, model interatomic potentials have found great utility in molecular dynamics simulations $[12,13,25-29]$. Although such potentials do not correspond to any specific real material, they help to elucidate the general principles governing crack tip behavior and fracture, such as "hyperelasticity" $[12,13]$.

Here, we use molecular dynamics simulations to investigate the influence of atomic structure and properties on the dynamic behavior of cracks in ductile materials. Specifically, 
we assess whether dynamics can suppress dislocation emission in a nominally ductile material, thereby promoting brittle cleavage, as proposed by Lin et al. [1]. To study this problem in a controlled manner, we employ a family of novel interatomic potentials for which the intrinsic ductility is tunable [30]. Specifically, for all potentials, the lattice constant, elastic constants, and fracture surface energy are held constant, so that the critical energy release rate for cleavage, $G_{I c}$, is fixed. However, the unstable stacking fault energy $\gamma_{u s}$ can be independently varied to tune the critical energy release rate for dislocation emission, $G_{I e}$. This allows us to conduct a "dynamic cleavage test" in which a crack initiates within a brittle material and runs dynamically into a second material (typically ductile). We find that dynamics cannot embrittle a ductile material; that is, if a crack tip at rest emits dislocations, then the same crack tip in motion will also emit dislocations. The origin of cleavage-like failure in mild steels at low temperatures is thus not due to a fundamental change in the competition between brittle and ductile crack tip behavior under dynamic conditions.

The remainder of this paper is organized as follows. In Section 2, we present the continuum analysis of dislocation emission and cleavage under quasistatic and dynamic conditions. In Section 3, we conduct molecular dynamics simulations of the dynamic cleavage test described above. In Section 4, we discuss our results further and propose alternative explanations for the observed brittleness of mild steels at low temperatures.

\section{Continuum analyses of dislocation emission and cleavage}

As has been widely discussed [14, 15], continuum-level analyses of crack tip deformation mechanisms such as cleavage and dislocation emission are not wrong, but simply incomplete. The first reason is that atoms play an important role in crack tip phenomena, such as lattice trapping (in quasi-static fracture) and forbidden steady-state velocities (in dynamic fracture). The second is that most continuum-level analyses consider only thermodynamics while neglecting kinetics. In dynamic fracture, many crack tip processes, including dislocation emission, cleavage, and phonon emission, may be thermodynamically possible, but all of these processes need not occur simultaneously. Instead, in such an "overloaded" state, kinetics-i.e., the complicated details of motion of atoms directly at the crack tip—will dictate which processes actually take place [26, 31]. Such details are often not amenable to analytical treatment, and thus the only recourse is numerical simulation. In spite of their deficiencies, continuum-level analyses remain useful because the thermodynamic criteria that they yield provide necessary, if not sufficient, conditions for crack tip deformation [7]. Two important examples are the Griffith criterion for brittle cleavage [32] and the Rice criterion for dislocation emission [6]. The competition between 
these two deformation mechanisms governs the intrinsic ductility of materials [6]. One powerful technique for deriving the Griffith and Rice criteria relies on path-independent crack tip contour integrals [33]. This method is convenient because it applies equally well to quasi-static and to dynamic steady-state cracks, as long as the appropriate crack tip integral is chosen.

We consider a crack in plane strain, with the crack plane normal in the $x_{2}$-direction. The crack propagates in the $x_{1}$-direction, and thus its crack front lies along the $x_{3}$-direction. Griffith cleavage in mode I occurs by the breaking of atomic bonds along the crack plane. The force law for tensile stretching of bonds is assumed to be given by $\sigma_{22}=f\left(\delta_{2}\right)$, where $\delta$ is the inelastic bond stretch. Using the quasistatic contour integral of Rice [34], and specializing the contour $(\Gamma)$ to the "process zone" within which all of the nonlinearity is confined, yields [34]

$$
J=\int_{\Gamma}\left[W n_{1}-n_{i} \sigma_{i j} \frac{\mathrm{d} u_{j}}{\mathrm{~d} x_{1}}\right] \mathrm{d} s=-\int_{\text {zone }} f\left(\delta_{2}\right) \frac{\mathrm{d} \delta_{2}}{\mathrm{~d} x_{1}} \mathrm{~d} x_{1}=\int_{0}^{\delta_{2, \max }} f\left(\delta_{2}\right) \mathrm{d} \delta_{2}
$$

since $n_{1}=0$ for the process zone. Since the integral of the atomic force law $f\left(\delta_{2}\right)$ is simply twice the surface energy, the Griffith criterion becomes

$$
G_{I c}=2 \gamma_{s}
$$

where $G$, which is identical to $J$ for linear elastic materials, characterizes the energy per unit area flowing towards the crack tip, and can be computed from the external loading. In exact analogy, dislocation emission in mode II can be predicted by considering slip in the $x_{1}$ direction along a process zone (the Peierls zone) normal to the $x_{2}$-direction. Slip is characterized a force-displacement relationship $\sigma_{12}=f\left(\delta_{1}\right)$. An essentially identical derivation [6] gives the result

$$
G_{I I e}=\gamma_{u s}
$$

where $\gamma_{u s}$ is the unstable stacking fault energy, which is the area under the positive part of $f\left(\delta_{1}\right)$. Thus, each criterion is couched in terms of a critical energy: $2 \gamma_{s}$ for the Griffith criterion and $\gamma_{u s}$ for the Rice criterion. Indeed, the ratio $\gamma_{s} / \gamma_{u s}$ characterizes the intrinsic ductility of the material [6].

For mode I dislocation emission along a slip plane oriented at some angle $\theta \neq 0$ with respect to the crack plane, the situation is more complicated and no exact analytical solutions are available. Rice [6] developed an approximate criterion for quasistatic mode I emission by assuming that emission occurs when a critical distribution of shear stresses is attained within the Peierls zone inclined at $\theta$. This distribution can be characterized by a single stress intensity factor $K_{\tau}$. In mode II, the critical value of $K_{\tau}$ is determined by computing the shear stresses at $\theta=0$ when $G=\gamma_{u s}$. In mode I, emission is assumed to 
occur when an identical $K_{\tau}$ exists along the plane oriented at $\theta$, yielding

$$
G_{I e}=\frac{8 \gamma_{u s}}{(1+\cos \theta) \sin ^{2} \theta}
$$

assuming that the material is isotropic and that the emitted dislocation lies parallel to the crack front [6].

We can straightforwardly extend the previous quasistatic analyses to the case of a dynamic, steady-state crack by using the dynamic path-independent integral $[35,36]$

$$
I=\int_{\Gamma}\left[(T+W) n_{1}-n_{i} \sigma_{i j} \frac{\mathrm{d} u_{j}}{\mathrm{~d} x_{1}}\right] \mathrm{d} s
$$

where $T$ is the kinetic energy density. I characterizes the energy flowing to the crack tip and thus equals $G$ for linear elastic materials [36]. Note, however, that for mode I cleavage and mode II emission, $n_{1}=0$, and so the kinetic energy term drops out of the analysis. Thus, these dynamic cases are identical to the quasistatic case: i.e., $G_{I c}=2 \gamma_{s}$ and $G_{I I e}=\gamma_{u s} \cdot{ }^{1}$

The approximate analysis of Rice for quasi-static mode I emission, based on stresses along the Peierls zone $\left(K_{\tau}\right)$, cannot be generalized to the dynamic case. For a steady-state dynamic crack loaded at a fixed $G$, all stress components decrease in magnitude as the velocity of the crack approaches the Rayleigh wave speed, $c_{R}$ [38]. In fact, as $v \rightarrow c_{R}$, the stresses become vanishingly small since all of the energy $G$ is converted into kinetic energy [38]. Thus, a stress-based analysis using $K_{\tau}$ would predict that dislocation emission would become highly unfavorable at large velocities, regardless of mode mixity. But this conclusion contradicts the exact analysis for mode II presented above. The contradiction arises because the stress-based approach assumes that only shear stresses drive dislocation emission while, in reality, both stresses and kinetic energy are important and the contribution of the latter is dominant at high velocities. Therefore, we suggest using the quasistatic $G_{I e}$ as the condition for emission from a mode I dynamic crack, just as $G_{I I e}$ is identical for both static and dynamic cracks. The atomistic results presented in Section 4 reveal that this condition works well.

The above continuum analyses for cleavage and dislocation emission based on pathindependent contour integrals indicate that the thermodynamic barriers for these processes are unaffected by dynamics. However, these analyses do not provide direct insight into the problem of key interest in this paper: notably, the "overloaded" situation when $G$ exceeds both $G_{I e}$ and $G_{I c}$. Such overloading is essentially forbidden for quasi-static cracks,

\footnotetext{
${ }^{1}$ Indeed, it is observed in molecular dynamics simulations of brittle fracture that cracks cannot propagate dynamically if $G<G_{I c}$. They may propagate at $G>G_{I c}$ if additional processes consume the excess energy [37].
} 
since deformation will occur when $G$ equals the lesser of $G_{I e}$ and $G_{I c}$. It can, however, occur for dynamic cracks crossing between materials, as in experiments and in the dynamic simulations presented below.

\section{Simulation of dynamic cleavage}

We conduct dynamic cleavage simulations in both 2D (hexagonal lattice) and 3D (FCC lattice) using molecular dynamics (MD). Although the 3D simulations are more computationally costly, they might also be more realistic: crack front variation in 3D might enable cracks to propagate when they would instead arrest in 2D. In other words, 2D materials might be artificially resistant to cleavage [15].

The simulation, illustrated schematically in Figure 1, uses a bi-material strip geometry. The strip geometry has two principal advantages with respect to loading $[15,26,31]$. First, a prescribed energy release rate, $G$, can be delivered to the crack tip to drive crack growth: it is readily demonstrated that $G=h u\left(\varepsilon_{22}\right)$, where $u\left(\varepsilon_{22}\right)$ is the strain energy density for uniform straining in the $x_{2}$-direction [15]. Second, the strip geometry enables steadystate crack growth, at a constant crack-tip velocity, using relatively small samples [15]. In steady state, the crack-tip stress fields can be computed analytically using standard expressions in dynamic fracture mechanics, and the contour integral analysis presented above is applicable [38].

The specimen is oriented with its crack front in the $x_{3}$-direction, and its crack plane perpendicular to the $x_{2}$-direction. The dimension of the specimen in the $x_{2}$-direction is $h$, where $h=356$ in 2D and 223 in 3D; these values are sufficiently large that the steady states should be approximately those associated with an infinitely tall specimen [15]; throughout this paper, all lengths are in units of the separation between nearest-neighbor atoms. We choose the specimen length in the $x_{1}$ direction, $L$, to be long enough $(L / h \geq 4)$ that the energy release rate is constant and that the crack reaches a steady-state velocity before encountering the interface. In the $2 \mathrm{D}$ simulation, the specimen length in the $x_{3}$-direction, $w$, is irrelevant. In the 3D simulations, we apply periodic boundary conditions in the $z$ direction with $w=45$; with this value, the specimen should be wide enough to allow dislocation loops to form [39], and to allow the crack front to vary over its length.

The boundary conditions are applied by fixing the positions of the top and bottom rows of atoms, and the system is loaded using the procedure described in Gumbsch et al. [26]. First, the specimen is stretched, by rescaling the atomic positions, to a strain corresponding to a $G$ slightly less than $G_{I c}$. The system is subsequently equilibrated using molecular 
statics so that the correct crack profile develops. Second, it is stretched again, to a $G$ slightly above $G_{I c}$, so that the crack begins to run. Finally, it is rescaled a third time to achieve the desired $G$. We also apply ramped viscous damping $[25,26]$ at the boundaries of the strip to mitigate the influence of reflected waves on crack motion. The simulations are essentially athermal, and we use the standard Velocity-Verlet algorithm with a timestep of 0.0018. The simulations are run using the Large-scale Atomic/Molecular Massively Parallel Simulator (LAMMPS) (Version June 28, 2014, Plimpton [40]), and the results are visualized using the Open Visualization Tool (OVITO) (Version 2.8.2, [41]). ${ }^{2}$

To describe interatomic interactions, we use a new flexible pair potential, developed for both 2D (hexagonal) crystals and 3D (FCC/HCP) crystals [30]. For these potentials, the unstable stacking fault energy can be tuned independently of all other relevant material properties. In other words, all materials studied here have the same lattice constant, elastic constants, and surface energy, and therefore they all have the same Griffith fracture energy, $G_{I c}$. The materials differ only in the value of $G_{I e}$, which can be tuned over a wide range, spanning the transition between brittle $\left(G_{I e}>G_{I c}\right)$ to ductile $\left(G_{I e}<G_{I c}\right)$ materials. In what follows, we refer to these potentials by their characteristic ratio of $G_{I e} / G_{I c}$, with larger values indicating greater brittleness. Because all near-equilibrium properties are the same, the bimaterial specimen is also elastically homogeneous and free of any residual stresses due to lattice mismatch; thus the effects of $G_{I e}$ can be probed independent of other complications.

In the $2 \mathrm{D}$ system, we start the crack in the most brittle material $\left(G_{I e} / G_{I c}=2.53\right)$. When propagating in this material, the crack remains atomically sharp, and is able to achieve a steady-state velocity for applied loads $G / G_{I c}$ less than roughly 1.8 . We then run the crack into the more-ductile materials having lower $G_{I e}$ and observe the behavior of the crack. The 3D FCC system is more challenging. Recall that the crack plane is a closepacked (111) plane and the crack front is [110]. This orientation is crystallographically unsymmetric about the crack plane, which is undesirable since purely tensile straining in the $x_{2}$-direction gives rise to mixed-mode loading in the strip geometry. Therefore, we symmetrize the system about $x_{2}$ by creating a symmetric twin boundary at $x_{2}=0$, and run the crack along this boundary (see Figure 5). In the 3D system, even the most brittle material emits before cleaving. To circumvent this problem, we alter the bonding along the putative crack plane (the twin boundary), thereby creating a weak interface that fails by atomically sharp cleavage. The elastic properties of the new bonds are the same, however, so the overall system remains elastically and crystallographically matched. The weak interface exists only in Material 1 (see Figure 1). We then run this crack into the more ductile 3D materials with lower $G_{I e}$. In both 2D and 3D, the interactions between

\footnotetext{
${ }^{2}$ We also post-process and visualize the data using a suite of Python tools, including NumPy (Version 1.8.1, [42]), Matplotlib (Version 1.3.1, [43]), and IPython (Version 0.12, [44]).
} 
atoms in Material 1 and Material 2 are given by the potential used in Material 1-the brittle potential. This "interface" interaction appears to be unimportant, however, since neither crack growth nor dislocation emission along the interface is observed. Note also that, since atomic masses and the elastic constants in the two materials are identical, no wave reflection arises at the interface, and the Rayleigh wave speed, $c_{R}$, is the same in both materials.

\section{Results}

\subsection{Dynamic cleavage in 2D}

In $2 D$, dynamic cleavage tests were run at two values of applied load: $G / G_{I c}=1.27$ and $G / G_{I c}=1.61$. The normalized crack velocity, $v / c_{R}$, vs. crack position is shown in Figure 2. ${ }^{3}$

At the lower loading of $G / G_{I c}=1.27$, dynamic cracks encountering the more-brittle materials $\left(G_{I e} / G_{I c}=1.72,2.10,2.53\right)$ grow dynamically into the second material while remaining atomically sharp, although the steady-state velocity may change. The velocity decreases with increasing $G_{I e}$, echoing previous findings that cracks in "softer," longerrange potentials typically attain lower steady-state velocities $[15,46]$. For these materials, in which $G<G_{I e}$, dislocation emission does not occur. The material with $G_{I e} / G_{I c}=1.37$ is a borderline case in this analysis. Thus, the critical energy release rate for dynamic dislocation emission appears to be identical to that in the quasistatic case, $G_{I e}$; dynamics does not affect the energetics of this process.

At this lower loading, dynamic cracks entering the more-ductile materials $\left(G_{I e} / G_{I c}=\right.$ $0.56,0.78,1.05)$, are overloaded since $G$ exceeds both $G_{I e}$ and $G_{I c}$. Therefore both dislocation emission and cleavage are energetically possible. The molecular dynamics simulations reveal that dislocation emission always occurs for overloaded cracks. And, once a single dislocation has been emitted, the competition between brittle and ductile behavior changes irrevocably. After emission, the crack rapidly slows to zero velocity, since it has essentially zero inertia and becomes blunted (see Figure 5(a)). Its subsequent behavior then depends on the critical quasistatic $G_{I e}$ and $G_{I c}$ in the blunted configuration [47]. For typical interatomic potentials, the crack tip radius $\rho$ does not affect the relative favorability of dislocation emission and brittle cleavage; if a sharp crack emits before cleaving, a blunted crack should as well [47]. Therefore, the crack continues to emit dislocations until

\footnotetext{
${ }^{3}$ Centrosymmetry [45] is used to locate the crack tip; the crack velocity is then computed by numerically differentiating the crack tip position with respect to time.
} 
$G_{I e}(\rho)$ drops below $G$. We conclude that all dynamic cracks running into ductile materials should arrest: materials that are quasistatically ductile are also dynamically ductile.

Identical conclusions emerge in studies at the higher overload of $G / G_{I c}=1.61$. At this load level, the case $G_{I e} / G_{I c}=1.72$ is borderline. In this case, the crack emits a single dislocation immediately upon entering the material, causing it to decelerate, but it subsequently manages to re-cleave and eventually reaches a steady-state velocity. For a higher overload of $G / G_{I c}=1.75$ (not shown), the crack emits more than one dislocation and arrests without re-cleaving, as expected since $G>G_{I e}$. Again, dislocation emission from dynamic cracks can be predicted reasonably well using the quasistatic Rice criterion, although borderline cases where $G \approx G_{I e}$ are not consistent.

In real materials, cracks typically propagate into ductile phases that are more compliant and denser than the brittle phases in which they originated. Recall that the crack speed scales with the Rayleigh wave speed, $c_{R} \propto \sqrt{\mu / \rho}$. Therefore, the "apparent" speed of the crack in the brittle material, as it just reaches the ductile material, may be large compared to $c_{R}$ of the ductile material ("relativistic"). We can explore these effects simply by altering the mass of the atoms in the second material, leaving the elastic constants unaffected. Here, we select $m_{2} / m_{1}=4$, so that the ratio of the Rayleigh wave speeds is $c_{R, 2} / c_{R, 1}=0.5$ and the crack enters Material 2 at a speed of nearly $c_{R, 2}$, well above the steady-state crack velocity in the material. Results for these "fast crack" simulations, with $G / G_{I c}=1.61$, are presented in Figure 3. As seen, cracks with $G<G_{I e}$ continue to propagate via cleavage, although their speed decreases drastically upon encountering the second material. Conversely, cracks with $G>G_{I e}$ emit dislocations, blunt, and arrest at the interface. Therefore, even when the crack velocities are relativistic, the quasistatic Rice criterion for dislocation emission works well and dislocation emission is kinetically preferred when the crack is overloaded.

We also explore effects of larger overloads, since brittle fracture in steels and other alloys usually involves energy release rates significantly in excess of the Griffith energy [1]. Marder [15] found that, in the thin strip geometry, highly-overloaded cracks blunt and arrest even in brittle materials, since no steady state can be attained. We have found that this arrest appears to be associated with wave reflections from the boundary. Although such reflections are of small amplitude, being heavily attenuated by the ramped viscous damping boundary conditions, they are sufficient to disrupt the unstable crack. We circumvent this issue by performing simulations in a much wider strip $(h=2670, L / h=4)$. For this size, waves emitted by the crack do not affect its subsequent propagation, since these waves do not have sufficient time to reflect off the boundary and interact with the crack. After an initial transient regime, the crack should be driven by a constant energy release rate and its velocity should attain an approximately constant value. At a large overload of 
$G / G_{I c}=4$, cracks in Material 1 (with $G_{I e} / G_{I c}=2.53$ ) emit dislocations as expected, but also simultaneously cleave. The cracks thus propagate unsteadily with velocities fluctuating around some average. Results are plotted in Figure 4, for times less than that needed for the reflected waves to return to the crack. Cracks in the more brittle materials (with $G_{I e} / G_{I c}=1.72,2.10,2.53$ ) propagate by simultaneous dislocation emission and cleavage. A similar mechanism was observed by Gumbsch [48]. On the other hand, cracks in the more ductile materials (with $G_{I e} / G_{I c}=0.56,0.78,1.05$ ) simply arrest directly at the interface. In the intermediate material with $G_{I e} / G_{I c}=1.37$, the crack cleaves over a short distance before blunting and arresting. There appears to be no simple criterion based on $G_{I e}$ and $G_{I c}$ for distinguishing between brittle and ductile fracture in this case, since even "brittle" fracture is accompanied by dislocation emission. However, $G_{I e}$ and $G_{I c}$ in the blunted configuration are likely important, since re-cleavage of the blunted crack must be possible for the brittle mode of failure to occur.

Every case reported above supports our main conclusion: materials that are quasi-statically ductile are also dynamically ductile. Dislocation emission occurs because its energy barrier is unaffected by dynamics and because it is evidently a kinetically favorable process when cleavage is also thermodynamically possible. Thus, dynamics appears not to be a contributing factor in material embrittlement at the atomistic crack tip.

\subsection{Dynamic cleavage in 3D}

The 3D FCC simulations yield similar results, and an identical conclusion: dynamic cracks emit dislocations if $G \gtrsim G_{I e}$, which causes them to blunt and arrest. For an applied load of $G / G_{I c}=1.39$, cracks run along the artificially-weakened interface in the first material and emit partial dislocations when they enter the second material, which has no weak interface. The emitted dislocations propagate away from the crack tip, leaving behind a faulted, HCP-like structure (Figure 5(b)). All cracks blunt and arrest (Figure 6(a)), even in the most brittle material $\left(G_{I e} / G_{I c}=1.66>G / G_{I c}\right)$. For a larger overload, $G / G_{I c}=2.47$ (not shown), all cracks again arrest upon reaching the second material. Note, however, that the crack dynamics are far more complicated at such a high overload, since the crack motion is unstable and many different mechanisms for energy dissipation can operate simultaneously [31].

The unexpected blunting of cracks in the FCC dynamic cleavage test may be related to the special geometry employed: that is, mirroring of the crystal using the twin boundary. The local elasticity may be slightly asymmetric, since the crack runs along one side of the twin boundary. This asymmetry leads to mode II loading, which can significantly promote dislocation emission even in small amounts [6]. Note, however, that in real materials, 
cracks running along $\{111\}$ planes do not run along special boundaries, and therefore these cracks experience a significantly greater component of mode II than in our simulations. Our test thus underestimates the ductility observed in a real crystal, which reinforces our conclusion that dynamics cannot embrittle a ductile material.

We have also run dynamic cleavage simulations in a special HCP orientation with a $(\overline{1010})$ crack plane and [1210] crack front. In this orientation, the loading is pure mode I, dislocations emit at $90^{\circ}$ to the crack plane, and both brittle and ductile fracture is possible in the pure material, without a weak interface. Results for an applied load of $G / G_{I c}=1.44$ with ductility ranging from $G_{I e} / G_{I c}=0.61$ to 1.92 are shown in Figure 6(b). As with the FCC and $2 \mathrm{D}$ systems, the crack remains sharp and cleaves through Material 2 when $G<G_{I e}$, whereas it emits dislocations, blunts, and arrests when $G>G_{I e}$.

We also fail to find any new effects associated with the 3D geometry. In 3D, the crack front may vary spatially, which means the crack can continue to propagate even if it happens to arrest locally. In the simulation, however, the crack front is observed to remain straight through the thickness of the specimen, as are the stacking faults left behind by the partial dislocations, even though the dislocation is likely nucleated from the crack tip as a loop [39]. Our examination of 3D effects in this study is somewhat limited, and so the conclusion that 3D effects are insignificant remains tentative. Like Marder [15], we believe that a more careful study of 3D effects, perhaps combined with effects of nonzero temperature, in brittle and ductile fracture is warranted.

\section{Discussion and conclusions}

Our two principal conclusions are as follows. First, the thermodynamics of dislocation emission are the same for static and dynamic cracks. This is exactly true for emission in Mode II, as demonstrated by the contour integral analysis, and appears to be approximately true for emission in Mode I, as demonstrated by the atomistic simulations. Second, if dislocation emission is thermodynamically possible, it is also kinetically favorable. That is, overloaded dynamic cracks entering a second material where $G>G_{I e}$, $G_{I c}$ will emit dislocations and subsequently blunt and arrest. Thus, there is no evidence that dynamic effects can embrittle a material, as hypothesized by Lin et al. [1], at least in terms of altering the local crack tip behavior.

Local blunting at the crack tip is not inconsistent with a nominally "brittle" low-toughness mode of fracture. Measured values of toughness in low temperature transgranular fracture are significantly larger than the Griffith energy [1], and fracture surfaces are not 
atomically flat. Thus, this failure mode is likely accompanied by substantial local plasticity. Fracture may then involve the interaction between the blunted crack tip and far-field dislocations, which may cause re-cleavage of the crack tip. The dynamic motion of dislocations emitted from the crack tip has been postulated as the mechanism for the brittleductile transition in Si [49] and a similar analysis was used by Hartmaier and Gumbsch [50] to explain semibrittle fracture in tungsten. These mechanisms are inherently largerscale phenomena, involving long-range dislocation interactions, and are thus not easily captured with atomistic methods alone. Multi-scale methods (e.g., Shilkrot et al. [51]) may therefore be able to resolve the disparities between experiments and atomistic simulations found in many material systems [52].

The findings of the present paper are consistent with the behavior in many material systems. In metal-matrix composites, such as $\mathrm{SiC} / \mathrm{Al}$, cracking of the brittle reinforcement does not embrittle the metal matrix and cause brittle fracture [53]. In fact, the deformation of the metal can be described reasonably well using a continuum plasticity model [53]. Blunting of cracks at the reinforcement/matrix interface has also been observed, using in situ microscopy, in Ti composites reinforced with TiB whiskers [54].

Finally, the experimental findings that originally motivated the hypothesis that, at low temperatures, brittle transgranular fracture can occur in mild steel remains unexplained. However, we can rationalize these experiments as follows. The $\alpha$-ferrite grains in mild steel are oriented randomly with respect to the dynamic cracks. In certain orientations, $G_{I e}$ is large, since dislocation emission needs to occur on planes that are oblique to the crack plane. In such orientations, the crack can cleave through these grains. Furthermore, ferrite is well-known to be quasi-ductile [47, 55], i.e. relatively low-toughness, and thus it is possible that local grain orientation can affect the delicate balance between emission and cleavage that exists in quasi-ductile materials. Cleavage of the overall material would then be a statistical process associated with the probability of finding grains favorably oriented for cleavage over emission, which is a different statistical argument than that proposed by Lin et al. [1]: notably, that the statistics of carbide strength and size control notched strength and toughness. One implication of our rationalization is that the toughness of steels may be improved through manipulation of the polycrystalline texture.

\section{Acknowledgements}

The authors gratefully acknowledge funding of this work through a European Research Council Advanced Grant, "Predictive Computational Metallurgy", ERC Grant agreement No. 339081-PreCoMet. The authors also thank Dr. Fabio Pavia for supplying the code 
for implementing ramped viscous damping in LAMMPS.

\section{References}

[1] T. Lin, A. G. Evans, and R. O. Ritchie. Stochastic modeling of the independent roles of particle size and grain size in transgranular cleavage fracture. Metallurgical Transactions A, 18(5):641-651, 1987.

[2] J. E. Hack, S. P. Chen, and D. J. Srolovitz. A kinetic criterion for quasi-brittle fracture. Acta Metallurgica, 37(7):1957-1970, 1989.

[3] B. N. Cox, H. Gao, D. Gross, and D. Rittel. Modern topics and challenges in dynamic fracture. Journal of the Mechanics and Physics of Solids, 53(3):565-596, 2005.

[4] I.-H. Lin and R. M. Thomson. Dynamic cleavage in ductile materials. Journal of Materials Research, 1(01):73-80, 1986.

[5] J. R. Rice and R. Thomson. Ductile versus brittle behaviour of crystals. Philosophical Magazine, 29(1):73-97, 1974.

[6] J. R. Rice. Dislocation nucleation from a crack tip: an analysis based on the Peierls concept. Journal of the Mechanics and Physics of Solids, 40(2):239-271, 1992.

[7] J. R. Rice. Thermodynamics of the quasi-static growth of Griffith cracks. Journal of the Mechanics and Physics of Solids, 26(2):61-78, 1978.

[8] W. A. Curtin. On lattice trapping of cracks. Journal of Materials Research, 5(07):15491560, 1990.

[9] R. Thomson, C. Hsieh, and V. Rana. Lattice trapping of fracture cracks. Journal of Applied Physics, 42(8):3154-3160, 1971.

[10] J. Fineberg, S. P. Gross, M. Marder, and H. L. Swinney. Instability in dynamic fracture. Physical Review Letters, 67(4):457, 1991.

[11] J. Fineberg and M. Marder. Instability in dynamic fracture. Physics Reports, 313(1): 1-108, 1999.

[12] M. J. Buehler, F. F. Abraham, and H. Gao. Hyperelasticity governs dynamic fracture at a critical length scale. Nature, 426(6963):141-146, 2003.

[13] M. J. Buehler and H. Gao. Dynamical fracture instabilities due to local hyperelasticity at crack tips. Nature, 439(7074):307-310, 2006. 
[14] M. Marder and S. Gross. Origin of crack tip instabilities. Journal of the Mechanics and Physics of Solids, 43(1):1-48, 1995.

[15] M. Marder. Effects of atoms on brittle fracture. International Journal of Fracture, 130 (2):517-555, 2004.

[16] J. Li, A. H. W. Ngan, and P. Gumbsch. Atomistic modeling of mechanical behavior. Acta Materialia, 51(19):5711-5742, 2003.

[17] Z. Wu and W. A. Curtin. Brittle and ductile crack-tip behavior in magnesium. Acta Materialia, 88:1-12, 2015.

[18] D. H. Warner and W. A. Curtin. Origins and implications of temperature-dependent activation energy barriers for dislocation nucleation in face-centered cubic metals. Acta Materialia, 57(14):4267-4277, 2009.

[19] R. Pérez and P. Gumbsch. Directional anisotropy in the cleavage fracture of silicon. Physical Review Letters, 84(23):5347, 2000.

[20] L. Hung and E. A. Carter. Ductile processes at aluminium crack tips: Comparison of orbital-free density functional theory with classical potential predictions. Modelling and Simulation in Materials Science and Engineering, 19(4):045002, 2011.

[21] D. Holland and M. Marder. Ideal brittle fracture of silicon studied with molecular dynamics. Physical Review Letters, 80(4):746-749, 1998.

[22] J. A. Hauch, D. Holland, M. P. Marder, and H. L. Swinney. Dynamic fracture in single crystal silicon. Physical Review Letters, 82(19):3823, 1999.

[23] J. R. Kermode, T. Albaret, D. Sherman, N. Bernstein, P. Gumbsch, M. C. Payne, G. Csányi, and A. De Vita. Low-speed fracture instabilities in a brittle crystal. Nature, 455(7217):1224-1227, 2008.

[24] M. Marder. Supersonic rupture of rubber. Journal of the Mechanics and Physics of Solids, 54(3):491-532, 2006.

[25] B. L. Holian and R. Ravelo. Fracture simulations using large-scale molecular dynamics. Physical Review B, 51(17):11275, 1995.

[26] P. Gumbsch, S. J. Zhou, and B. L. Holian. Molecular dynamics investigation of dynamic crack stability. Physical Review B, 55(6):3445, 1997.

[27] S. J. Zhou, P. S. Lomdahl, A. F. Voter, and B. L. Holian. Three-dimensional fracture via large-scale molecular dynamics. Engineering Fracture Mechanics, 61(1):173-187, 1998. 
[28] F. F. Abraham, D. Brodbeck, W. E. Rudge, J. Q. Broughton, D. Schneider, B. Land, D. Lifka, J. Gerner, M. Rosenkrantz, J. Skovira, and H. Gao. Ab initio dynamics of rapid fracture. Modelling and Simulation in Materials Science and Engineering, 6(5):639670, 1998.

[29] F. F. Abraham, R. Walkup, H. Gao, M. Duchaineau, T. D. De La Rubia, and M. Seager. Simulating materials failure by using up to one billion atoms and the world's fastest computer: Work-hardening. Proceedings of the National Academy of Sciences, 99(9): 5783-5787, 2002.

[30] V. P. Rajan, D. H. Warner, and W. A. Curtin. A novel interatomic potential with tunable intrinsic ductility. Submitted, Modelling and Simulation in Materials Science and Engineering.

[31] P. Gumbsch. On the response of dynamic cracks to increasing overload. In MRS Proceedings, volume 409, page 297. Cambridge Univ Press, 1995.

[32] A. A. Griffith. The phenomena of rupture and flow in solids. Philosophical Transactions of the Royal Society of London, Series A, pages 163-198, 1921.

[33] B. Moran and C. F. Shih. A general treatment of crack tip contour integrals. International Journal of Fracture, 35(4):295-310, 1987.

[34] J. R. Rice. A path independent integral and the approximate analysis of strain concentration by notches and cracks. Journal of Applied Mechanics, 35(2):379-386, 1968.

[35] C. Atkinson and J. D. Eshelby. The flow of energy into the tip of a moving crack. International Journal of Fracture Mechanics, 4(1):3-8, 1968.

[36] L. B. Freund and J. W. Hutchinson. High strain-rate crack growth in rate-dependent plastic solids. Journal of the Mechanics and Physics of Solids, 33(2):169-191, 1985.

[37] B. L. Holian and R. Thomson. Crack limiting velocity. Physical Review E, 56(1):1071$1079,1997$.

[38] L. B. Freund. Dynamic Fracture Mechanics. Cambridge University Press, 1998.

[39] T. Zhu, J. Li, and S. Yip. Atomistic study of dislocation loop emission from a crack tip. Physical Review Letters, 93(2):025503, 2004.

[40] S. Plimpton. Fast parallel algorithms for short-range molecular dynamics. Journal of Computational Physics, 117(1):1-19, 1995.

[41] A. Stukowski. Visualization and analysis of atomistic simulation data with OVITOthe open visualization tool. Modelling and Simulation in Materials Science and Engineering, 18(1):015012, 2010. 
[42] S. Van Der Walt, S. C. Colbert, and G. Varoquaux. The NumPy array: a structure for efficient numerical computation. Computing in Science E Engineering, 13(2):22-30, 2011.

[43] J. D. Hunter. Matplotlib: A 2D graphics environment. Computing in Science and Engineering, 9(3):90-95, 2007.

[44] F. Pérez and B. E. Granger. IPython: a system for interactive scientific computing. Computing in Science and Engineering, 9(3):21-29, 2007.

[45] C. L. Kelchner, S. J. Plimpton, and J. C. Hamilton. Dislocation nucleation and defect structure during surface indentation. Physical Review B, 58(17):11085, 1998.

[46] B. L. Holian, R. Blumenfeld, and P. Gumbsch. An Einstein model of brittle crack propagation. Physical Review Letters, 78(1):78-81, 1997.

[47] G. E. Beltz, D. M. Lipkin, and L. L. Fischer. Role of crack blunting in ductile versus brittle response of crystalline materials. Physical Review Letters, 82(22):4468-4471, 1999.

[48] P. Gumbsch. An atomistic study of brittle fracture: Toward explicit failure criteria from atomistic modeling. Journal of Materials Research, 10(11):2897-2907, 1995.

[49] P. B. Hirsch and S. G. Roberts. The brittle-ductile transition in silicon. Philosophical Magazine A, 64(1):55-80, 1991.

[50] A. Hartmaier and P. Gumbsch. Thermal activation of crack-tip plasticity: The brittle or ductile response of a stationary crack loaded to failure. Physical Review B, 71(2): 024108, 2005.

[51] L. E. Shilkrot, R. E. Miller, and W. A. Curtin. Multiscale plasticity modeling: Coupled atomistics and discrete dislocation mechanics. Journal of the Mechanics and Physics of Solids, 52(4):755-787, 2004.

[52] K. Ravi-Chandar. Dynamic fracture of nominally brittle materials. International Journal of Fracture, 90(1-2):83-102, 1998.

[53] M. T. Kiser, F. W. Zok, and D. S. Wilkinson. Plastic flow and fracture of a particulate metal matrix composite. Acta Materialia, 44(9):3465-3476, 1996.

[54] C. J. Boehlert, C. J. Cowen, S. Tamirisakandala, D. J. McEldowney, and D. B. Miracle. In situ scanning electron microscopy observations of tensile deformation in a boronmodified Ti-6Al-4V alloy. Scripta Materialia, 55(5):465-468, 2006.

[55] V. Shastry and D. Farkas. Molecular statics simulation of fracture in $\alpha$-iron. Modelling and Simulation in Materials Science and Engineering, 4(5):473-492, 1996. 


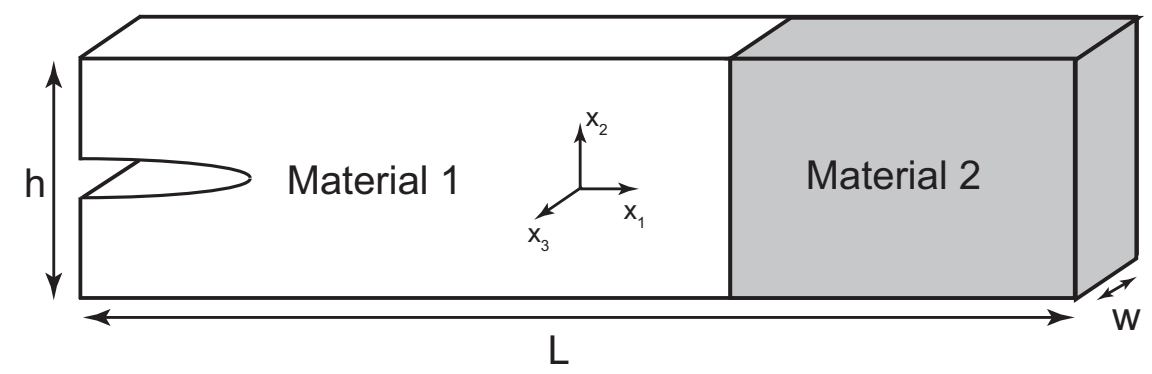

Figure 1: Strip geometry for dynamic cleavage test.

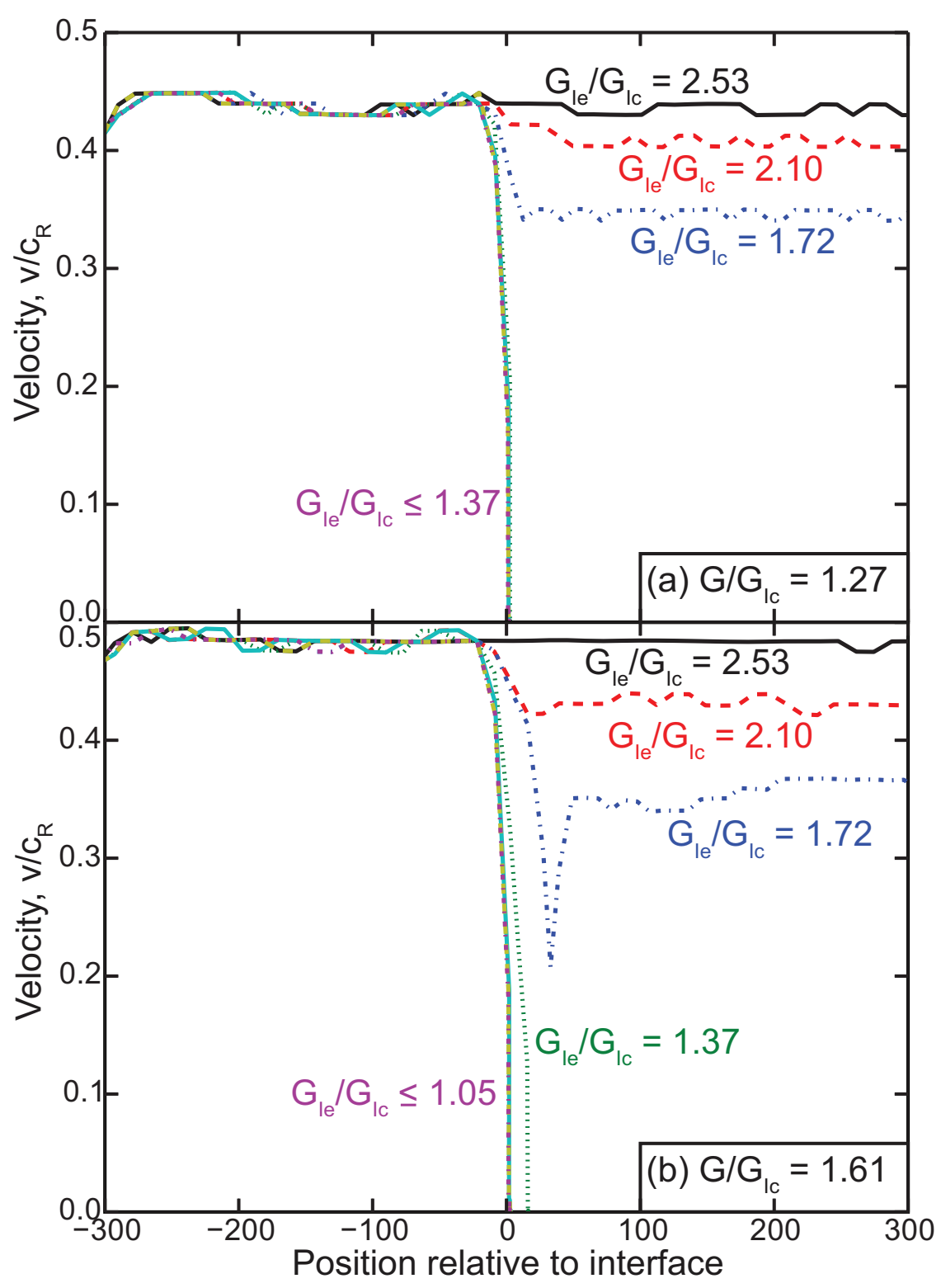

Figure 2: Crack velocity in 2D dynamic cleavage test. The second material has varying ductility $\left(G_{I e} / G_{I c}=0.56-2.53\right)$. Two values of applied load: (a) $G / G_{I c}=1.27$ and (b) $G / G_{I c}=1.61$. 


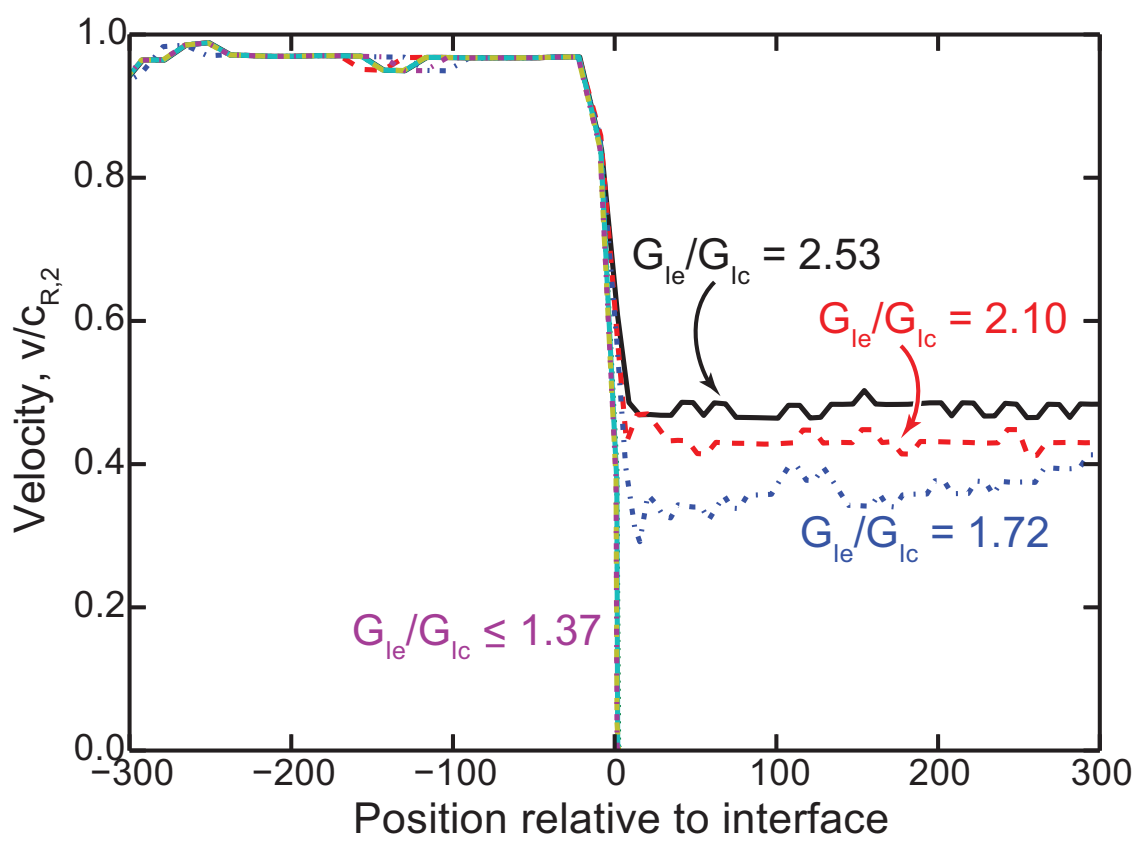

Figure 3: Crack velocity in 2D dynamic cleavage test, with materials of different mass: $m_{2} / m_{1}=4$, so that the ratio of the Rayleigh wave speeds is $c_{R, 2} / c_{R, 1}=0.5$. The second material has varying ductility $\left(G_{I e} / G_{I c}=0.56-2.53\right)$. The applied load is $G / G_{I c}=1.61$, and crack velocities are normalized by $c_{R, 2}$.

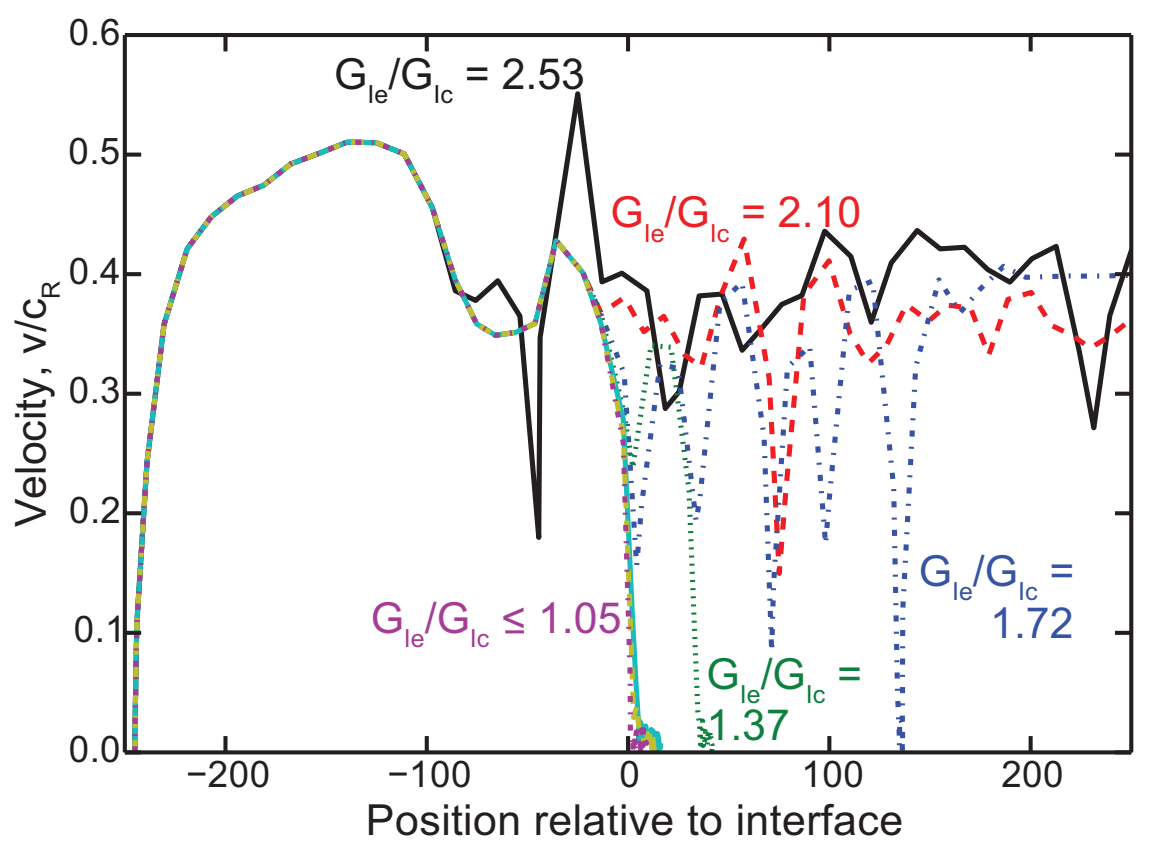

Figure 4: Crack velocity in a highly overloaded $2 \mathrm{D}$ dynamic cleavage test $\left(G / G_{I c}=4\right)$. The second material has varying ductility $\left(G_{I e} / G_{I c}=0.56-2.53\right)$. 
This is a pre-print of the following article: Rajan, V. P.; Curtin, W. A. J. Mech. Phys. Solids 2016, 90, 18-28.. The formal publication is available at http://dx.doi.org/10.1016/j.jmps.2016.02.014
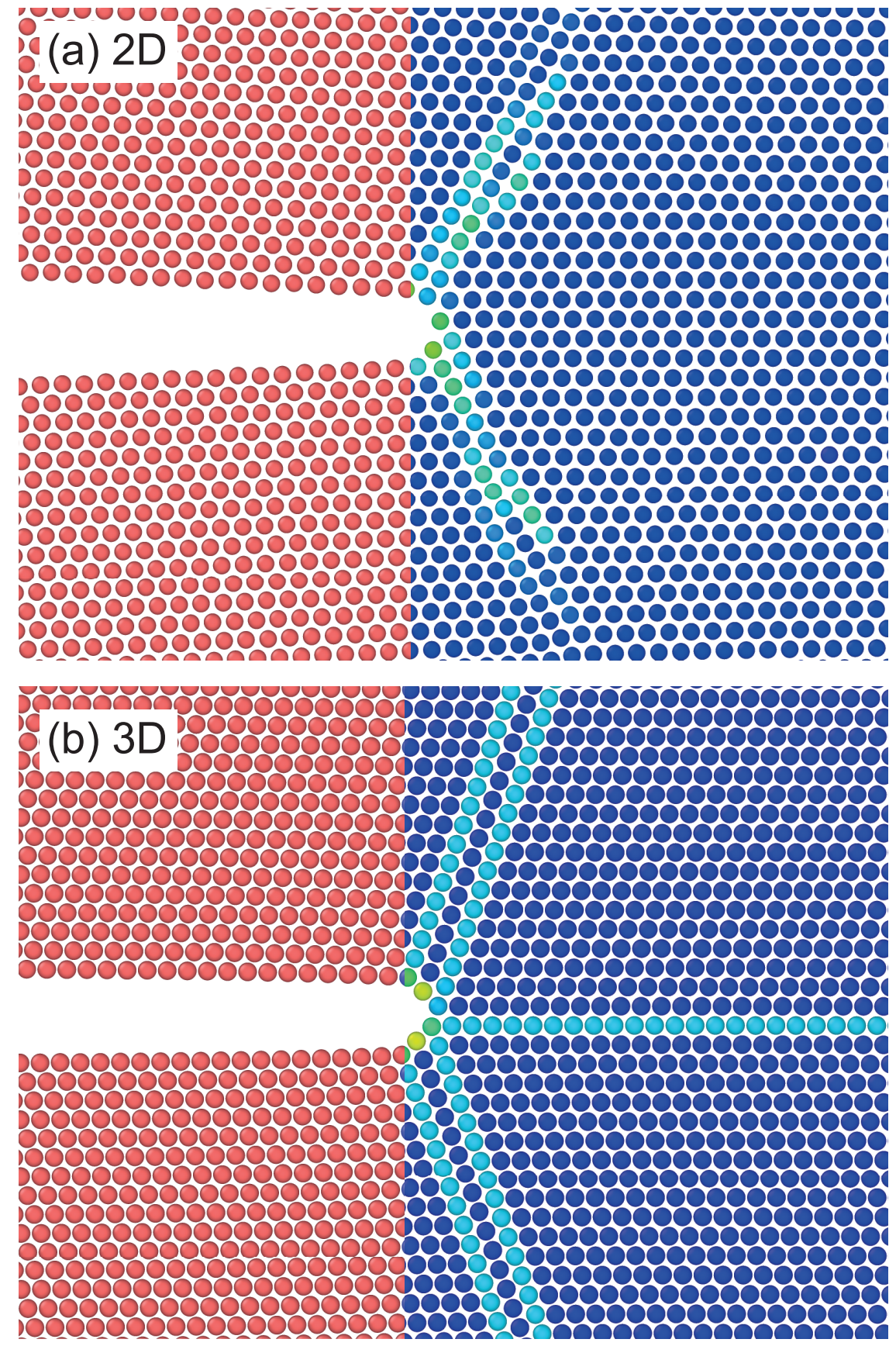

Figure 5: Profiles of blunted cracks, just after reaching the interface in the dynamic cleavage test, in (a) $2 \mathrm{D}\left(G / G_{I c}=1.27\right)$ and (b) $3 \mathrm{D}$ FCC $\left(G / G_{I c}=1.39\right)$. For both tests, the second material corresponds to the most ductile potential; atoms in the second material are colored according to their centrosymmetry, which reveals dislocations/stacking faults. 


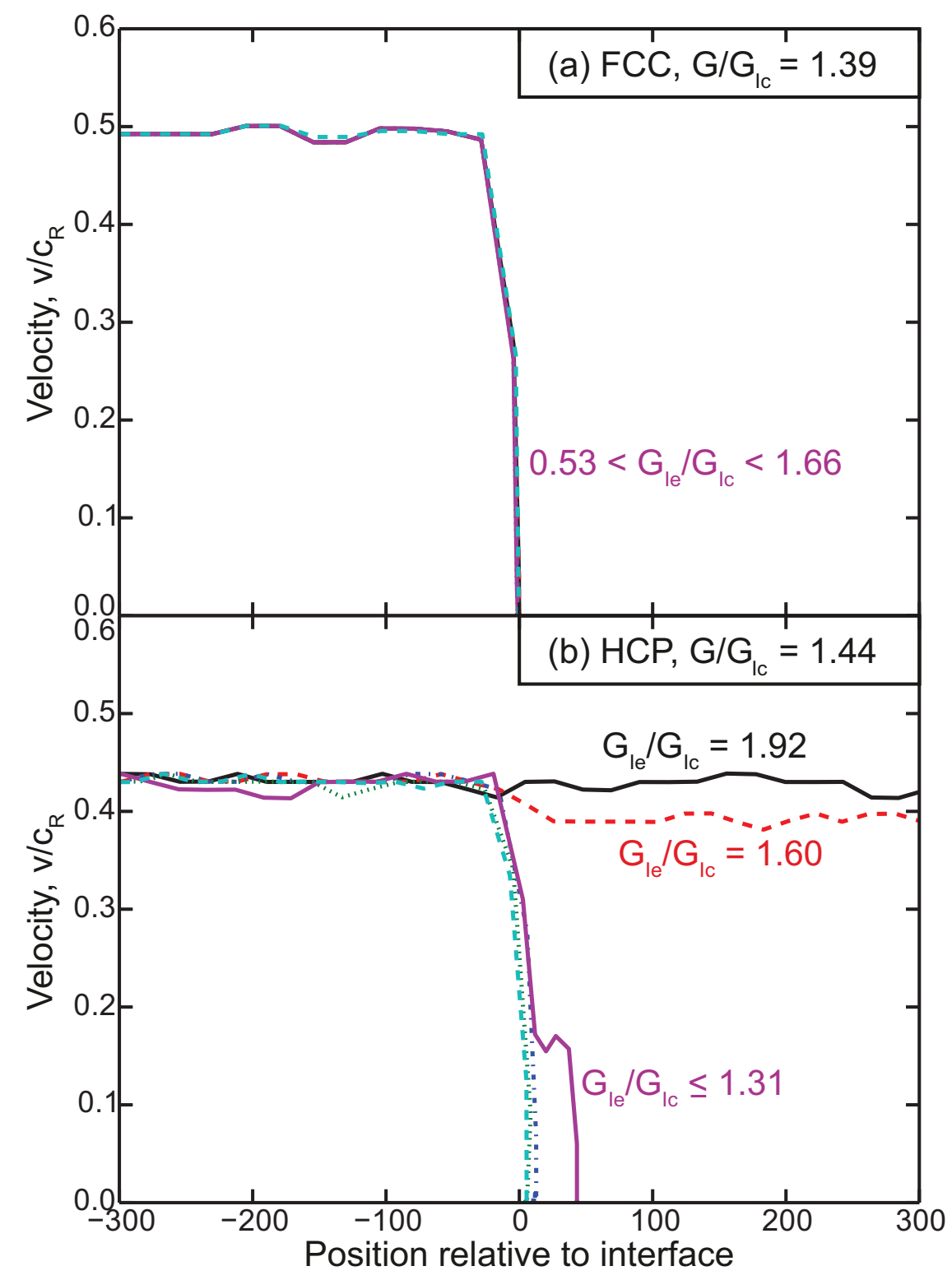

Figure 6: Crack velocity in 3D dynamic cleavage test. The second material has varying ductility $\left(G_{I e} / G_{I c}=0.53-1.66\right)$. In the FCC system (a), the applied load is $G / G_{I c}=1.39$; in the HCP system (b), $G / G_{I c}=1.44$. 